\title{
Italique
}

Poésie italienne de la Renaissance

XX $\mid 2017$

Varia

\section{Riprese strutturali della bucolica classica nel Quattrocento}

Maria Finazzi

\section{OpenEdition}

Journals

Edizione digitale

URL: http://journals.openedition.org/italique/452

DOI: $10.4000 /$ italique.452

ISSN: 1663-4438

Editore

Librairie Droz

\section{Edizione cartacea}

Data di pubblicazione: 1 octobre 2017

Paginazione: 47-72

ISBN: 978-2-600-05818-6

ISSN: 1423-3983

Notizia bibliografica digitale

Maria Finazzi, «Riprese strutturali della bucolica classica nel Quattrocento », Italique [Online], XX I

2017, online dal 01 octobre 2019, consultato il 10 décembre 2020. URL : http://

journals.openedition.org/italique/452 ; DOI : https://doi.org/10.4000/italique.452

(c) Tous droits réservés 


$$
\text { MAR I A Fin A Z Z I }
$$

R I PRESE STRUTTURALI D E L L A B C O L I C A C A S I C A NEL Q U A T R O C E N T O 



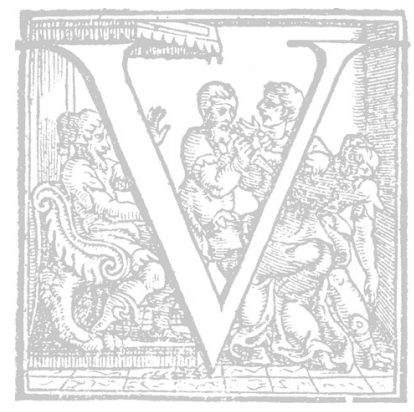

no studio delle riprese della bucolica classica (in particolare virgiliana) nelle egloghe volgari del Quattrocento, se condotto con particolare attenzione alla struttura e alla distribuzione degli argomenti allinterno dei testi, consente di evidenziarne la matrice pastorale più di quanto sia stato fatto finora, e comunque di rafforzare rapporti intertestuali già acquisiti sulla base di altri riscontri. Lo studio dell'argomento non può che partire dal modello classico per eccellenza, le egloghe di Virgilio. Queste presentano, a livello micro- e macrotestuale, precise caratteristiche strutturali di suddivisione e distribuzione della materia, e mostrano fra l'altro una notevole attenzione compositiva al piano per cosi dire quantitativo (cioè al numero totale di versi del testo e delle sue porzioni): mi riferisco al fatto che, com'è noto, «incredibilmente la somma del numero dei versi di [alcune] coppie [di egloghe] dà risultati corrispondenti: ecl. I e ecl. 9: I50 versi $(83+67)$; ecl. 2 e ecl. 8: I8I versi $(73+$ I08); ecl. 3 e ecl. 7: I8I versi $(I I I+70)$; ecl. 4 e ecl. 6: I49 versi $(63+86) »$ e che «le egloghe $1-4$ e 6-9 abbracciano un numero uguale diversi $; ; "$ e che tale «simmetria regna anche all'interno delle singole egloghe», ad es. con prologhi ed epiloghi della medesima estensione ( $5 v v$. in $\mathrm{ViBu} I$ ) e coppie di canti aventi la stessa lunghezza (in $\mathrm{ViBu}$ s e $\mathrm{ViBu}$ 8: rispettivamente di 25 e $45 \mathrm{vv}$.).

Si possono intanto proporre alcune osservazioni preliminari sul macrotesto. Nel liber virgiliano è stata individuata una precisa volontà ordinatrice che guida la successione e la rispondenza delle egloghe tra di loro: un'ipotesi di lettura (che si avvale appunto di corrispondenze numeriche nella somma dei versi delle egloghe) vede le prime 9 egloghe disposte simmetricamente a cornice, secondo criteri tematici e/ o formali, attorno alla 5 , che fa da centro dell'opera (lo schema degli accoppiamenti è dunque: I/9, 2/8, 3/7, 4/6), e con la Io posta come conclusione; un'altra ipotesi vede l'opera costituita da due serie, I-4 e 6-9, ciascuna tendente a un culmine, rispettivamente s e Io. Come sottolineato tra gli altri da Stefano Carrai, l'autore bucolico quattrocentesco che più si è contraddistinto per la «fedeltà verso il modello classico del libro pasto-

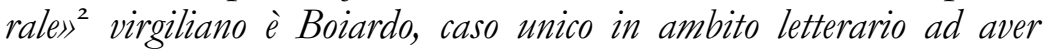
prodotto, com'è noto, sia una serie di Io bucoliche in lingua latina, sia una serie di ro bucoliche in lingua volgare. Nella serie latina dei $\mathrm{Pa}-$ 
storalia la «sistematica emulazione» del modello «fa si che ogni egloga risponda in tutto o in parte alla corrispettiva virgiliana) ${ }^{3}$ (con riscontri sia contenutistici che strutturali nelle prime sei e solo strutturali nelle altre quattro). Nella serie volgare delle Pastorale Carrai rileva anche lo sforzo "di dare coesione all' insieme raccordando i singoli testi mediante richiami lessicali e tematici tra il finale dell' uno e l'inizio del successivo» ${ }^{4}$ (procedimento tipico «del genere canzoniere»). ${ }^{5}$ Per altri autori entrano invece in gioco modelli diversi, come le I2 egloghe del Bucolicum carmen di Petrarca. Queste, come ha evidenziato Francesca Battera, agiscono sulle 8 egloghe del Benivieni, ${ }^{6}$ e hanno probabilmente avuto un ruolo nella decisione di Sannazaro di portare l'Arcadia da Io a I2 prose/egloghe. Passando al livello microtestuale, va sottolineato come l'individuazione della struttura all'interno di un singolo testo non sia sempre operazione scontata. Le difficoltà si debbono a due motivi principali: in primo luogo nel testo si sourappongono, a volte senza precise corrispondenze, diversi ordini di struttura (per fare l'esempio più semplice, nei testi dialogati la suddivisione delle battute tra i personaggi non sempre coincide con la suddivisione tematica); in secondo luogo, diversi possono essere i punti di vista da cui si guarda alle strutture interne al testo: dalla situazione narrativa o dalla forma del contenuto (cioè dal modo in cui $i$ contenuti vengono presentati: incontro e dialogo tra pastori, racconto del canto/dei canti di pastori) dipende infatti la presenza di uno o più livelli e/o forme del discorso (dialogo, narrazione, discorsi diretti o indiretti riportati). Quando i livelli siano almeno due (uno narrativo e uno drammatico), la loro combinazione può dar luogo a strutturazioni chiuse (è il caso della cornice, cioè presenza di prologo e congedo a uno o più canti) o a strutturazioni aperte (quando è presente solo il prologo, cioè un'introduzione narrativa, e il testo si conclude con la messa in scena della porzione parlata/dialogata). Possono darsi inoltre casi di diverse letture possibili e ugualmente plausibili: ad esempio $\mathrm{ViBu} 6$ e, più interessante per il discorso che andremo a fare, ViBu 2. Come sottolinea Massimo Gioseffi nella sua edizione commentata delle Bucoliche di Virgilio, ${ }^{7}$ i vv. 74-8I di ViBu 6, che fanno parte del canto di Titiro, possono essere interpretati in diversi modi: non è chiaro se, all'interno del canto di Titiro, costituiscano una seconda serie di miti metamorfici da includere nel riassunto del canto di Sileno oppure fungano da riassunto dei canti di Gallo; aggiungo che, portando all'estremo la lettera del testo, von Albrecht, in una noticina alla sua analisi delle opere 
virgiliane, correggendo un altro studioso, sottolinea come i versi del canto di Sileno «sono cantati dalle Muse, non, come egli crede, da Titiro» ${ }^{8}$ (una interpretazione molto letterale di "Pergite, Pierides» al v. I3). ${ }^{9}$ Ancora secondo Gioseffi in ViBu 2 (l'egloga che contiene il lamento di Coridone) il testo che segue i primi s vv. di prologo (dove la voce di un io narrante introduce la figura del pastore) tollera ugualmente bene due ipotesi di partizione e attribuzione della materia, la prima a struttura aperta, la seconda chiusa: ${ }^{\text {10 }}$

I) nella prima interpretazione il monologo di Coridone arriva fino alle fine del testo (raggiungendo cosi la lunghezza di $68 \mathrm{vv}$.), secondo lo schema $5+68$;

2) nella seconda interpretazione il monologo si interrompe al $v .68$ (fermandosi alla lunghezza di $63 \mathrm{vv}$.), lasciando i s vv. finali all'io narrante, in perfetta simmetria con il prologo di s $\mathrm{v}$., secondo lo schema $5+63+s:$ struttura vicinissima a quella del modello TeId II, l'idillio del Ciclope, al quale Virgilio aggiunge però la misura simmetrica dei due elementi della cornice (la formula che rappresenta la struttura teocritea è $18+62+2$, con al centro il canto/lamento di Polifemo).

Esiste inoltre una terza lettura, proposta da Luca Canali nella sua edizione commentata delle Bucoliche, che pur se erronea torna utile per evidenziare che si tratta di questioni insidiose: in questa interpretazione il monologo di Coridone si interrompe al v. 5) (raggiungendo la lunghezza di $50 \mathrm{vv}$.) e $i$ restanti is $\mathrm{vv}$. vengono attribuiti alla voce narrante, secondo lo schema $5+50+i 8$ (tale interpretazione è erronea visto che al v. 58 torna la prima persona: "quid volui misero mibi?»). $V a$ da sé che la molteplicità di letture possibili deve essere tenuta presente anche quando si riflette sul ruolo di modello svolto da questi testi su autori successivi: casopercaso andrà infatti valutato in che modo il poeta quattrocentesco leggeva i suoi auctores. ${ }^{\text {I }}$ Nel verificare l'eventuale ripresa di un modello, con un occhio all'equilibrio proporzionale/quantitativo tra le diverse parti, non va trascurata, inoltre, l'ipotesi che siano stati necessari adattamenti di natura metrica. Prendo come esempio il volgarizzamento delle Bucoliche di Virgilio realizzato da Bernardo Pulci, concentrandomi su un'area fortemente strutturata come è il canto amebeo, su cui tornerò tra breve: $i$ due amebei virgiliani sono composti uno, $\mathrm{ViBu} 3$, da coppie di distici, l'altro, $\mathrm{ViBu} 7$, da coppie di quartine; in entrambi $i$ casi Pulci, che ha scelto come forma metrica il capitolo in terzine, è 
costretto a un'amplificazione del testo, per far rientrare ogni battuta nella misura di una o più terzine, risolvendo il primo caso con coppie di $3 \mathrm{vv}$., il secondo con coppie di $6 \mathrm{vv}$.

La struttura delle bucoliche virgiliane è fondata sulla variazione di un insieme piuttosto ristretto di elementi. ${ }^{\mathrm{I}}$ Su questo insieme di elementi giocheranno ulteriormente anche Calpurnio e Nemesiano (che, ricordo, fino al sec. XV sono stati tramandati insieme e considerati un unico autore), introducendo nuove variazioni come la gara che alla fine non si svolge di CaEc 6.

Vorrei ora prendere in considerazione due particolari tipologie di egloghe: lo scambio di canti tra due pastori e il canto di Polifemo (promosso a vero e proprio sottogenere bucolico), passando poi ad analizzare alcune riprese di queste tipologie nella bucolica quattrocentesca.

\section{LO SCAMBIO DI CANTI}

Nelle Bucoliche di Virgilio gli scambi di canti tra pastori sono in tutto quattro, due amebei e due non-amebei. Il primo scambio di canti amebei è senza cornice narrativa $(\mathrm{ViBu} 3)$ : nella prima parte $(54 \mathrm{vv}$ ) $i$ pastori dialogano scambiandosi reciproche accuse, si sfidano e coinvolgono un terzo pastore come giudice; nella seconda parte ba luogo la gara (uno scambio di canti amebei, I2 coppie di distici su 6 diversi temi, totale $48 \mathrm{vv}$.), introdotta e conclusa dal gindice $(5+48+4=$ totale 57 $v v$.). Il secondo scambio di canti amebei ba una cornice narrativa drammatizzata, attribuita come abbiamo visto al pastore Melibeo e non a un generico io narrante ( $\mathrm{ViBu} 7$ ): Melibeo racconta di essere stato chiamato da Dafni ad assistere alla gara tra Coridone e Tirsi, che poi viene riportata subito di seguito con il discorso diretto ( 6 coppie di quartine, totale $48 \mathrm{vv}$ ); nell'epilogo di $2 \mathrm{vv}$. è sancita la vittoria di Coridone. Anche il primo scambio di canti non-amebei è senza cornice narrativa ( $\mathrm{ViBu} 5): i$ due canti di $25 \mathrm{v} v$. dedicati rispettivamente alla morte e all'apoteosi di Dafni sono preceduti, inframmezzati e seguiti da battute di dialogo dei due pastori protagonisti, Menalca (che poi canta per secondo) e Mopso (cui spetta il primo canto). Il secondo scambio di canti non-amebei è costruito su due livelli a struttura aperta (ViBu 8): ${ }^{3}$ l'io narrante introduce l'argomento dell'egloga (la gara tra $i$ due pastori), dedica il testo a un illustre e anonimo protettore e descrive il momento della gara (all'alba, un momento insolito), e poi torna con un 
breve intermezzo di raccordo tra $i$ due canti; $i$ pastori, Damone e Alfesibeo, si scambiano due canti entrambi della lunghezza di $45 \mathrm{vv}$. (se si espunge il $v .76$ dal secondo canto) e con verso intercalare, il primo che dà voce a un amante infelice che ba perso la sua Nisa e per questo medita il suicidio, il secondo che dà voce a una donna che, compiendo un rito magico, cerca di far ritornare l'amato Dafni dalla città.

Veniamo ora alle possibili riprese.

Alla tipologia dello scambio amebeo ci riporta quello che viene considerato il più bucolico dei testi egloghistici di Leon Battista Alberti, il Tyrsis (AlTy). Si tratta di un'egloga dialogica, con due interlocutori (Floro e Tirsi) che prima parlano tra loro epoi si scambiano canti amebei, e in cui è presente anche un piano diegetico: nei primi versi l'autore introduce i due pastori e descrive l'occasione del dialogo. La lunghezza del testo dell'Alberti (88) si colloca fra quella di $\mathrm{NeEc} I(87)$ e quella di $\mathrm{NeEc} 2 e$ ViBu s (90). Gorni, in AlBERTI2002, p. I9I, notacheè lastessa lunghezza dei capitoli dell'Amorosa visione di Boccaccio. Metricamente abbiamo di fronte un testo in terzine, chiuso da una quartina di schema XYYX (la rima $X$ è anche al secondo verso dell'ultima terzina). ${ }^{\mathrm{I}}{ }^{4}$ Vediamo il testo dall'inizio. Nelle prime due terzine l'autore introduce $i$ due pastori e descrive l'occasione del dialogo (6 vv.); segue un dialogo, con una terzina per battuta, in cui i due pastori confrontano le loro situazioni amorose: mentre Tirsi è innamorato felice di Mea, Floro è innamorato infelice di Niera, e per consolarsi viene invitato da Tirsi al canto (I2); quest'ultimo pronuncia una breve canzone pastorale di due terzine, a cui Floro risponde, a tema, con una battuta di due terzine (I2: 6+6); Tirsi rinnova il suo invito al canto (b); segue un canto amebeo a strofe dispari, iniziato e concluso dallo stesso interlocutore (Floro): le prime due battute (con l'apostrofe a selve e ninfe, e ad altri elementi naturali insieme) occupano lo spazio di due terzine, le successive II battute sono di una terzina ciascuna, sull'ingiustizia delle sofferenze amorose e sulla superbia di chi resiste ad amore (45, cosi scandito: $6+6,3+3,3,3+3,3+3,3+3,3+3)$; in conclusione l'autore (sub voce Phaunorum?) apostrofa $i$ due giovani pastori e chiude l'egloga con una riflessione sentenziosa sulla natura amorosa delle donne.

Il testo presenta in effetti diverse particolarità strutturali che illustro in sintesi:

a) ripetuta alternanza di parti dialogate e scambi di canto, secondo questo schema (in cui le parti amebee sembrerebbero essere due, anche se 
in alcuni luoghi il passaggio dal dialogo al canto amebeo avviene all'interno della terzina o del singolo verso - ad es. al v.37 - con una modalità di transižione un po' opaca):

- prologo narrativo (2t)

- dialogo: scambio di battute sulle rispettive amate e invito al pianto (da parte di Floro) o invito al canto (da parte di Tirsi) (4t)

- canto amebeo $2 \mathrm{t}+2 \mathrm{t}$

- dialogo: nuovo invito al canto (sempre da parte di Tirsi) (2t)

- canto amebeo $2 \mathrm{t}+2 \mathrm{t}$ e poi $\mathrm{It}+\mathrm{It}$ (dispari)

- epilogo (narratore o phauni?);

b) il canto amebeo presenta partizioni di diversa lunghezza (prima di $2 t / 6 v v$. e poi di $\mathrm{It} / 3 v v$ );

c) il canto amebeo è asimmetrico, a strofe dispari (la serie di scambi di It inizia e si conclude con Floro; in realtà a restare irrelata è la terza terinina della serie - XIX nel testo, vv. 55-57);

d) a livello narrativo sembra cambiare il punto di vista: nel prologo troviamo un generico io narrante; nell'epilogo, a seconda delle interpretazioni (anche qui strutturazione chiusa o strutturazione aperta), ricorre di nuovo un generico io narrante che apostrofa $i$ due protagonisti oppure compare un terzo interlocutore (per di più collettivo).

La maggior parte di queste caratteristiche strutturali sono riconducibili a diversi esempi classici:

a) ritroviamo l'ambiguità di statuto tra dialogo e canto amebeo, e cioè una certa opacità circa il momento in cui avviene il passaggio dal mero dialogo al canto o al canto amebeo vero e proprio in $\mathrm{CaEc}$ 2;

b) dallo stesso testo calpurniano deriva l'altra particolarità dell'amebeo adottata dall'Alberti, e cioè la diversa lunghezza delle strofe: in CaEc 2 infatti $i$ $70 \mathrm{vv}$. dello scambio amebeo fra Ida e Astaco è formato da I6 strofe di $4 \mathrm{vv}$. ciascuna più due strofe finali di $3 \mathrm{vv}$. ciascuna;

c) per l'adozione di un amebeo a strofe dispari si può risalire all'influsso di $\mathrm{CaEc} 4$; è questo un esempio di come un'anomalia dovuta a corruzione della tradizione testuale (per l'egloga di Calpurnio la caduta della quarta strofa amebea, da cui è derivata una scorretta attribuzione delle strofe successive ai due interlocutori) che non viene rilevata in quanto tale dai lettori successivi, dà luogo a un nuovo fenomeno testuale (l'ame- 
beo a strofe dispari); se nel testo di Calpurnio è infatti possibile riassegnare le strofe a quello che doveva essere l'interlocutore originario, questo non può accadere nell'egloga dell'Alberti (dove, se non altro, è lo schema delle rime a garantire della successione dei $v v$.); la seconda battuta di Floro della misura di una terzina (vv. 55-57) resta "scompagnata", senza nessuna risposta da parte di Tirsi, che, dalla battuta successiva, diventa quindi il proponente del tema; per inciso, le strofe amebee successive al secondo invito al canto da parte di Tirsi assommano in tutto 45 vv., una misura come ho già indicato "virgiliana" (anche se Virgilio la adotta non negli amebei, ma per l'intera lunghezza di ciascuno dei due canti di $\mathrm{ViBu} 8$ );

d) non ho per ora ritrovato precedenti per l'anomalia del finale (sempre che quest'ultima battuta sia davvero da riferire ai Phauni).

Detto ciò, mi pare che il modello alla base dell'ideazione di questo testo sia $\mathrm{NeEc} 4$ (verso intercalare e struttura aperta a parte), che, insieme a $\mathrm{CaEc} 2$ e NeEc 2, è tra le egloghe classiche (già sinteticamente elencate da Tissoni Benvenuti 1978, p. 1079) in cui due pastori innamorati confrontano $i$ rispettivi amori. Questi sono gli elementi comuni ai due testi:

i) attacco del testo (al v. 2 «pastores [...] uterque» > «pastori l'uno e l'altro», al v. 3 《proprios cantabat» > «racontar suoi canti»);

ii) tipologia di interlocutori (due pastori);

iii) tipologia di occasione del canto (scambio in un momento di riposo);

iv) temi, immagini e riprese lessicali (in particolare nell'attacco e nella parte amebea) che si susseguono quasi nella stessa successione nei due testi:

Nemesiano, Ecl. IV

A. vv. 2-3 pastores, calamis et versu doctus uterque

nec triviale sonans, proprios cantabat amores.

B. vv. 26 e $33-34$

Mop. Cerva marem sequitur, taurum formosa invenca

[...]

Ver erat, et vitulos vidi sub matribus istos,

qui nunc pro nivea coiere in cornua vacca.

C. vv. $27-28$

et Venerem sensere lupae, sensere leaenae et genus aerium volucres et squamea turba D. v. 50

Mop. Qui tulerit Meroes fastidia lenta superbae,

E. v. 60

si modo sollicitos aliquis deus audit amantes.
L.B. Alberti, Tyrsis

richi pastori, l'uno e l'altro bello, usi fra loro racontar suoi canti,

B. vv. 2 I e 28

che hai quelli occhiazzi più bei che'l mio toro,

[...]

come un giovenco mal uso ad arare

E. vv. 45 e 49

Audissi Amor chi lui cantando onora.

[...]

Flo. Se Amor è iusto e pio, come'l permette

D. vv. 57-60

ancora è più superba la mia Niera.

Tyr. Dura, ostinata è chi non amassi: sogionsi, cantan li augeleti amando; aman le fiere, gli orsi, lupi e tassi. 
Ai luoghi sopra evidenziati se ne possono aggiungere altri due o tre, che non riporto, in cui ritroviamo gli stessi concetti ma con modalità espressive e lessicali diverse. Anticipo inoltre che, a mio parere, la stessa modalità di richiamarsi alla fonte principale del testo riprendendone proprio l'attacco è messa in atto dall'Alberti anche nel Corymbus. E significativo che i rimandi - anche solo lessicali - alla quarta egloga di Nemesiano si interrompano ai due terzi del testo albertiano: si può dire che, per la conclusione della propria egloga, Alberti rifiuta in blocco la conclusione nemesianea, in cui si accenna al (bucolico) ricorso a pratiche magiche, per lasciare spazio a ulteriori riflessioni sulla precettistica amorosa (in piena sintonia con analoghe riflessioni condotte dall' Alberti in altri amatoria). Inoltre, stante la componente precettistica sull'amore che scandisce tutta l'egloga e che si concentra nella parte finale, ${ }^{\mathrm{IS}}$ non stupisce rinvenire numerosi riscontri da Properzio e altri elegiaci (Tibullo e Ovidio) - in linea con la lettura delle opere dell'Alberti offerta nei suoi studi da Roberto Cardini. ${ }^{1}{ }^{6}$

Un paio di testi della Pastorale di Boiardo appartenenti alla tipologia dello scambio di canti portano a osservare quanto l'occhio di Boiardo fosse attento alle misure virgiliane per quanto riguarda la lunghezza dei singoli canti e la proporzione tra le parti dialogiche e le parti di canto amebeo. Nel primo esempio, BoPE 4, i pastori Melibeo e Dameta nel momento di riposo del mezzogiorno decidono di scambiarsi canti con intercalare. L'egloga, un capitolo in terzine di I6g vv., può essere cosi suddivisa:

- vV. I-45 ( 15 t. $/ 45$ vV.): i due pastori si accordano per scambiarsi un canto-lamento (in sintonia con la tristezza del momento storico - il riferimento è alla cattura di Niccolò da Correggio da parte dei veneziani nel I 482); inizia Dameta con un canto che «Pur mo’ compose e scrisse in verde foglia» (v. 27);

- vv. 46-9o ( 15 t./45 vv.): canto di Dameta: prigionia di Teseo;

- vv. 9I-IOS ( 5 t./ I 5 vv.): Melibeo intende rispondere con un canto che ha «composto cum scorza di fagio» (v. $9^{8}$ );

- vv. ro6-I 50 ( 15 t./45 vv.): canto di Melibeo: profezia della liberazione di Teseo;

- vv. I 5 I-I 69 (6 t. e I V./I 9 Vv., suddivisibili in $5 \mathrm{t}$. + I t. e I v./I $5+4$ vV.): i pastori commentano la veridicità della profezia, e si congedano. 
Lo schema generale è quello di ViBu s (già ripreso da Boiardo per BoPA 5), una cornice con didascalia intermedia a racchiudere $i$ due canti, di pari lunghezza; cosi come appartiene a $\mathrm{ViBu}$ s la continuità tematica tra $i$ due canti (in Virgilio la morte e l'apoteosi di Dafni, in Boiardo la prigionia e - sotto forma di profezia - la liberazione di Teseo/Niccolò da Correggio). Inoltre, $i$ due canti dell'egloga di Boiardo sono caratterizzati dalla presenza del verso intercalare, come i due canti di ViBu 8 (la Pharmaceutria); e, su quest'ultima egloga virgiliana pare essere commisurata anche la loro lunghezza di $45 \mathrm{vv}{ }^{17}$

Nel secondo esempio, BoPE 7, assistiamo alla lite tra due pastori Damone e Gorgo, e alla successiva gara di canto amebeo arbitrata da Corina, fanciulla amata da Gorgo. Come nella raccolta latina, Boiardo colloca la gara amebea nella seconda posizione scelta da Virgilio per una gara amebea $(\mathrm{ViBu} 7)$, riprendendo però le modalità del racconto e la struttura del canto dalla prima gara amebea di Virgilio ( $\mathrm{ViBu}$ 3): il testo è diviso in due parti, la prima in cui $i$ due pastori contendenti prima litigano e poi si accordano per una gara di canto; la seconda in cui si svolge la gara vera e propria, con l'intervento del giudice che apre e chiude la gara, facendo da cornice allo scambio di amebei, cosi:

a) Vv. I-48 (48 vv.): lite, sfida e scelta del giudice

b) vv. 49-57 (9 vv.): il giudice dà avvio alla gara

vv. 58-i I I ( $54 \mathrm{vv}$.): canto amebeo (9 coppie di terzine)

vv. I I 2-27 (I6 vv.): il giudice decreta il pareggio

Nel suo recente commento Cristina Montagnani sottolinea come «la settima egloga [...] riprende [dopo la quinta egloga] e conclude il serrato confronto con gli esperimenti di Francesco Arzocchi, e assieme segna un punto di riallineamento con le serie latine $[\mathrm{BoPA} 7 \mathrm{e} \mathrm{ViBU} 7$ contengono due gare amebee]», e poi aggiunge che «in qualche tratto è anche evidente nel testo volgare una connessione con Buc. III, che tradizionalmente fa coppia con VII: penso ad esempio al registro comico, che appare più marcato in Boiardo che nella VII delle Bucoliche, ed è invece assai rilevato nella III. Inoltre la gara resta senza conclusione in PA VII e in PE VII come in Buc. III, mentre in Buc. VII è Coridone a riportare il successo». ${ }^{18}$ Ora, tanto più alla luce di queste osservazioni, non credo sia un caso che la proporzione tra le due componenti dell'egloga (lite e preparativi della gara vs gara vera e 
propria), sia la stessa di $\mathrm{ViBu}$ 3, a parti invertite: nella bucolica di Virgilio (che misura III vv.) la prima sezione occupa $54 \mathrm{vv}$. (suddivisibili in: $6+2 I$ contrasto e accuse; 27 sfida e preparativi) mentre il canto amebeo vero e proprio misura $48 \mathrm{vv}$. (I2 coppie di distici), incorniciato dai due interventi del gindice rispettivamente di s $\mathrm{v}$. prima e $4 \mathrm{vv}$. dopo:

Virgilio, Buc. III

Prima parte: 54

Seconda parte: $5+48+4$
Boiardo, Pastorale VII

Prima parte: 48

Seconda parte: $9+54+{ }_{16} 6$

\section{IL “CANTo di Polifemo”}

Passando ora al sottogenere bucolico del canto di Polifemo, inizio col notare che la struttura dei testi classici è varia:

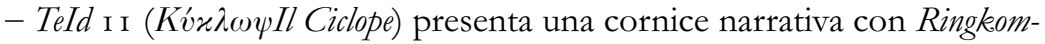
position a racchiudere un monologo, secondo lo schema: $18+62+2$; il monologo/lamento/canto di Polifemo è inserito all'interno di una cornice (in cui il poeta si rivolge a un interlocutore esterno, il medico/poeta Nicia) con Ringkomposition (l'amore/ '̌ $\varrho \omega \tau \alpha$ che ricorre nell'incipit e nel penultimo verso del testo, entrambi appartententi alla cornice); la chiusa di 2 vv. ribadisce il ruolo della poesia come rimedio d'amore;

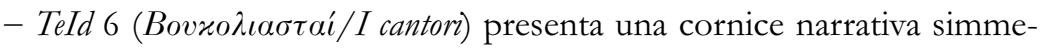
trica a racchiudere lo scambio di due canti di diversa lunghezza con un verso intermedio, secondo lo schema $5+\mathrm{I}_{4}+_{\mathrm{I}}+{ }_{20}+5$; l'amore di Polifemo per Galatea è narrato attraverso lo scambio di canti tra due pastori (Dafni e Dameta), il primo (di I 4 vv.) sotto forma di apostrofe al ciclope e il secondo (di 20 vv.) sotto forma di monologo di costui (il tutto all'interno di una cornice simmetrica di 5 vv. iniziali e 5 finali, più I v. di raccordo tra i due canti, per un totale di $45 \mathrm{vv}$. (e non 46 , espungendo il v. 4I dal testo e dal secondo canto); la chiusa sancisce lapidariamente che «Nessuno vinse, nessuno fu sconfitto»;

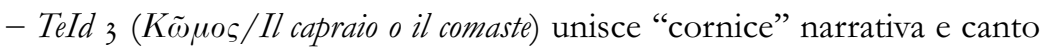
sotto la stessa voce: è il capraio infatti a introdurre se stesso in atto di cantare prima una serenata per Amarilli e poi un canto mitologico, intervallati da una parentesi narrativa, e, infine, a raccontare l'interruzione del suo cantare, secondo lo schema $5+30+3+{ }_{2}+3$.

Ho già descritto le due possibili strutturazioni (aperta o chiusa) di $\mathrm{ViBu}$ 2; aggiungo solo che il monologo di Coridone è suddiviso in blocchi scanditi da esclamazioni e vocativi (rispettivamente di II, II, I7, 
II, I3 e, in una delle due possibili letture, $5 v v$. finali); la chiusa del testo, «invenies alium [...] Alexin» ("troverai un altro Alessi»), suggerendo una facile consolazione, apre per dirla con von Albrecht al «disincanto». ${ }^{19}$

Anche nei testi volgari che ora analizzerò brevemente il contenuto è strutturato in modo vario. Il primo testo è il Corymbus di Leon Battista Alberti (AlCo). Il lamento del pastore albertiano presenta una strutturazione metrica basata sulla simmetria: Is $\mathrm{vv}$. in terzine $(s t)+.{ }_{I}$ ballata di $44 v v .+15 v v$. in terzine $\left(5\right.$ t.), per un totale di $74 v v{ }^{2 \circ}$ Del lamento del pastore albertiano si è spesso sottostimata la natura bucolica. Tra gli elementi che vari critici e commentatori (tra gli altri Gorni e Tanturli) hanno trovato poco bucolici troviamo:

- la maggiore indeterminatezza del testo albertiano rispetto ai precedenti classici (Coridone si identifica subito come «pastor», a differenza del «giovinetto» Corimbo)

- l'assenza di elementi specificamente pastorali (ben presenti invece sia nelle cornici narrative sia nei monologhi di TeId i e ViBu 2);

- la definizione di «morale» attribuitagli insieme alla Mirtia, come abbiamo già visto, nel ms. $\mathrm{F}_{10}{ }^{21}$

- il fatto che i due personaggi in realtà non dialogano realmente, ma si alternano semplicemente.

Per rispondere a queste perplessità si possono fare diverse osservazioni. Innanzitutto il modello virgiliano $\mathrm{ViBu} 2 \mathrm{e}$ il testo dell' Alberti hanno quasi la stessa lunghez?a: $73 \mathrm{vv}$. in Virgilio, $74 \mathrm{vv}$. in Alberti. Da questo punto di vista, la scelta albertiana di inserire una ballata di endecasillabi e settenari nella parte centrale del testo (riducendo lo spazio testuale a disposizione, tanto più che la lingua volgare è meno sintetica della lingua latina e quindi a parità di contenuto richiederebbe piu spazio) ha costretto l'autore a limitare il contenuto, a favore della parte elegiaco/didascalica. Prima di procedere è il caso di affrontare una questione che riguarda l'interpunzione e l'impaginazione (e quindi l'interpretazione) del testo: nelle sue due edizioni delle rime dell' Alberti Gorni ha impaginato il testo come un'egloga dialogata, attribuendo $i$ due blocchi simmetrici di s terzine, e cioè i primi is e gli ultimi is versi, al personaggio Battista, e la sola ballata al personaggio Corimbo (sulla scorta delle rubriche degli interlocutori in $F_{I o}$ dove, a quanto riporta 
Grayson in ALBERTI 1966, p. 400, «accanto aivv. I e 60 si legge M. B. (Messer Battista), e al v. I6 Corimbo»; nel primo blocco di terzine le parole fatte pronunciare a Corimbo (che inizia con «Infelice chi inciampa»e finisce con «mio dolore entro prega, e di fuor tace») sono inserite in un discorso diretto riportato tra virgolette all'interno della battuta di Messer Battista:

- al v. 7: Spesso «Infelice» dicea «chi inciampa

- al v. I5: mio dolore entro prega, e di fuor tace».

Per giustificare questa citazione di un discorso all'interno di una battuta di dialogo ("citazione che Batista fa dei versi di Corimbo (7-I5)») Gorni si rifà alla fonte virgiliana $\mathrm{ViBu}$ 9, in cui «Lycidas e Moeris» si fanno «cantori [...] del carmen di Menalca»). ${ }^{22}$ In realtà il modello scelto dall'Alberti, secondo me, è un altro, e, come nel Tyrsis, è dichiarato dall'autore attraverso il calco di alcune espressioni nei primi $v v$. del componimento:

\begin{tabular}{|c|c|}
\hline $\begin{array}{l}\text { Nemesiano, Ecl. IV I-3 } \\
\text { Populea Lycidas nec non et Mopsus in umbra } \\
\text { pastores, calamis et versu doctus uterque } \\
\text { nec triviale sonans, proprios cantabat amores. }\end{array}$ & $\begin{array}{l}\text { L.B. Alberti, Tyrsis I-3 } \\
\text { Tyrsis e Floro, gioveneti amanti, } \\
\text { richi pastori, l'uno e l'altro bello, } \\
\text { usi fra loro racontar suoi canti, }\end{array}$ \\
\hline $\begin{array}{l}\text { Virgilio, Buc. II I-5 } \\
\text { Formosum pastor Corydon ardebat Alexin, } \\
\text { delicias domini: nec quid speraret habebat. } \\
\text { Tantum inter densas, umbrosa cacumina, fagos } \\
\text { adsidue veniebat; ibi haec incondita solus } \\
\text { montibus et silvis studio iactabat inani: }\end{array}$ & $\begin{array}{l}\text { L.B. Alberti, Corymbus I-6 } \\
\text { Corimbo, giovinetto averniese, } \\
\text { bello, prudente, virtuoso, onesto, } \\
\text { in cui eran d'Amor le faci incese, } \\
\text { di selva in selva giva solo, mesto, } \\
\text { spegnendo con le lagrime la vampa, } \\
\text { qual a sé stesso lo rendeva infesto. }\end{array}$ \\
\hline
\end{tabular}

Il riferimento è ovviamente a $\mathrm{ViBu} 2$, con uno slittamento (forse non casuale come dirò poi) per cui in Virgilio Formosus ("formose puen》 al v. I7) è il giovane Alexis, che rifugge l'amore di Coridone, mentre in Alberti è l'innamorato Corimbo a essere «giovinetto [...] bello».

Con un occhio a quest'altro modello virgiliano (il lamento di un pastore riportato all'interno di una narrazione, a struttura chiusa o aperta a seconda delle interpretazioni del testo) è però possibile pensare a una seconda possibilità di impaginazione/interpunzione (che è presente anche nell'edizione ALBERTI I97 I curata da Folena), che fa iniziare il discorso diretto di Corimbo al v. 7 ("Spesso, "Infelice”, dicia, "chi inciampa») e lo prosegue senza soluzione di continuità fin verso la fine del testo (vedremo tra un momento dove precisamente); non siamo quindi di fronte a un'egloga dialogata (o paradialogata), bensi a un 
testo in cui l'io narrante riporta, sotto forma di discorso diretto, il lamento del protagonista. Il problema di dove far finire il discorso diretto ci porta a riproporre le diverse possibili interpretazioni del testo della fonte latina (e della relativa fonte greca, TeId $I I$ ).

Ora, anche la parte finale del testo albertiano (vv. 60-74, cioè le s terz̨ine finali) si potrebbe prestare a diverse letture, o, comunque, è suddivisibile in due parti distinte per tono e destinatario: le prime 4 terzine sono rivolte infatti a "Corimbo», usando la seconda persona singolare, $e$ contengono una serie di apostrofi incalzanti e di inviti a rassegnarsi al dominio di Amore; la terzina finale è invece rivolta ai «miseri amanti», usando la seconda persona plurale, e il tono si fa più distaccato. Tenendo a mente le due fonti del testo (in prima battuta quella virgiliana, $e$ in seconda battuta quella teocritea), e stante il mancato accordo dei due testimoni sulle didascalie, mi pare siano possibili tre distribuzioni di queste terzine:

I. nella prima i is versi finali sarebbero da attribuire al narratore (come nell'edizione Gorni), dando luogo a una struttura a cornice: $6+59+$ I 5 ; 2. nella seconda le prime 4 terzine della conclusione potrebbero essere interrogative retoriche che Corimbo rivolge a se stesso, e solo l'ultima terzina toccherebbe al narratore, con una struttura a cornice $6+65+3$ rapportabile agli esempi classici di TeId in (18+62+2) e ViBu 2 (nell'interpretazione a struttura chiusa secondo lo schema $5+63+5$ );

3. nella terza i is versi finali farebbero parte del monologo di Corimbo (come nell'ed. Folena), con una struttura aperta $6+68$ rapportabile al modello virgiliano $V i B u 2$ (nell'interpretazione con schema $5+68$ ).

Che il rapporto strutturale tra il testo albertiano e la fonte virgiliana sia stretto si ricava anche dalla misura dei due testi e delle possibilipartizioni interne. Anzi, proprio l'eventuale aderenza al modello virgiliano farebbe propendere per le scansioni $6+6806+65+3$; mentre la prima soluzione $(6+59+15)$ mi pare si debba considerare, insieme alla piu simmetrica Is $\left.+44+\operatorname{~IS~(del~ms.~} F_{I 0}\right)$, come erronea, dovuta a una falsa suddivisione delle battute ricalcata sulle partizioni metriche (e, nel secondo caso, influenzata dall'idea di una ripresa della simmetria virgiliana del $5+63+5)$. Quanto agli altri presunti elementi "extrabucolici": anche nei testi virgiliani i pastori sono più volte indicati come semplici pueri. ${ }^{23}$ Inoltre, come notava Tissoni Benvenuti in una recensione all'ed. Gorni, 
considero egloga (non «elegia dialogata» come vorrebbe il Gorni) anche il Corimbo: sia perché esemplato sulla II di Virgilio sia perché nell'attenta onomastica dell'Alberti - acutamente studiata dallo stesso Gorni per la Mirtia, donna del mirto, in opposizione a lauro/Laura - Corimbo può ricordare il parallelo Coridone (come sarà antenato del Corinto mediceo), ma impone soprattutto al lettore l'immediata associazione ai corimbi dell'edera: e corone d'edera, non d'alloro incoronavano secondo la tradizione classica i poeti pastori. ${ }^{24}$

In conclusione, il testo di Alberti è chiaramente costruito ricalcando la struttura di $\mathrm{ViBu} 2$, con numerose riprese concentrate, per dirla con De Robertis, nel «preambolo didascalico» e nell' «intervento ammonitorio finale» (elementi «del Tyrsis» che il Corimbo «ripete»); ${ }^{25}$ queste riprese a volte sono solo lessicali, magari con cambiamento di riferimento o significato finale, come il formosum/bello che in Alberti è riferito all'amante e non all'amato, o il crudelis/crudel che in Alberti è più in generale Amore e non l'amato. Ma è anche da questi slittamenti che assume evidenza il possibile senso dell'operazione di Alberti: introdurre nell'egloga non un'esperienza personalizzata, bensi un più generico discorso su/ad Amore, non una vicenda dettagliata nel quotidiano bensi una figura di innamorato più universale, cui applicare quella precettistica elaborata poi negli altri amatoria in prosa (Deifira, in primis). Non a caso l'unico riferimento all'oggetto dell'amore di Corimbo è l'acrostico MARIA, segnalato per la prima volta da Gorni nella ri-edizione francese delle rime dell'Alberti, ${ }^{26}$ che si ricava dalle lettere iniziali delle stanze della ballata. Diverso, come nel caso di AlTy in confronto alla fonte nemesianea, il senso della conclusione: Virgilio invita a passare a un altro amore, Alberti invita a non desistere.

Appartengono al sottogenere del "Canto di Polifemo" due egloghe di Boiardo, BoPE 2 e BoPE 9. La seconda delle Pastorale di Boiardo, in cui come da didascalia "parla lo auttor e canta Galathea», adotta una struttura chiusa: prologo di 2 I vv, canto di Galatea di $102 \mathrm{vv}$., ed epilogo di $7 \mathrm{vv}$. Con variatio rispetto ai precedenti classici, il poeta («autton》 nella rubrica) in veste di pastore è in loco ed è lui ad essere «tacito e pensoso» mentre si va «a posare in su la riva» del Po (vv. I-2), ed è testimone dell'emergere della ninfa dalle acque del fiume e ascoltatore delle «parole» mosse «a guisa de lamento» (v. 2I). Come evidenziato dai commentatori delle Pastorale il testo si distacca dall'omologa 
virgiliana. D'altro canto, materia e struttura del "classico" lamento di Coridone vengono con maggior evidenza adottati da Boiardo nella simmetrica BoPE $9^{27}$ in cui «lamentasi cantando Corydone per la sua cara Nysa a Mopso maritata». In quest'ultima notiamo che è assente la cornice $^{28}$ e siamo di fronte a un'egloga interamente monodica. ${ }^{29} E$ cosi in Boiardo i versi finali sono sicuramente una autoapostrofe di Coridone, a cui va attribuita anche la massima generale rivolta agli amanti. Il testo è comunque suddivisibile in tre parti, che ricalcano la struttura di $\mathrm{ViBu} 2$, solo che prologo ed epilogo (che nel modello virgiliano sono enunciati da un narratore esterno) qui sono tutti affidati alla voce di Coridone:

I. apostrofe del pastore agli elementi naturali del locus amoenus (che svolge la funzione di definire l'ambientazione, nel modello affidata al prologo); 2. canto di Coridone in cui sono presenti elementi di altre bucoliche virgiliane ( $V i B u$ 8, in particolare nel primo canto di Damone, e ViBu 3); 3. uscita di Coridone dal canto, momento metapoetico (vv. 76-77 e 82: «Ma lasso, ché vagando io son trascorso / e del passato parlo di presente $/[$...] / Nel mio cantare è persa ogni misura»), fino al calco virgiliano del v. 85 «Ah, Coridone, ove hai tanta folia?» che richiama in maniera forte la chiusa di ViBu 2, con l'apostrofe di Coridone a se stesso, con il "tu" (non fosse per l'assenza di un prologo narrativo) interpretabile anche come apostrofe del narratore allo "stolto" pastore protagonista del lamento e come dispensa di insegnamento generale agli amanti: «mal fa chi per altrui sé stesso perde», v. 94.

Lorenzo de Medici quindicenne sceglie di esordire in ambito bucolico con un canto di Polifemo, il Corinto, che già dal titolo sembrerebbe rinviare al Corimbo albertiano $\mathrm{e} / \mathrm{o}$ al Coridone virgiliano. ${ }^{30} \mathrm{La}$ misura del testo è notevolmente diversa rispetto alle bucoliche classiche: la prima redazione dell'egloga laurenziana, ${ }^{3 \mathrm{I}}$ di soli $175 \mathrm{vv}$. (contro $i$ I84 della definitiva), è già due volte e mezzo $\mathrm{ViBu} 2$ (73 vv.). Come è caratteristico di Lorenそo, nel testo vengono ripresi una varietà di autori classici (mi rifaccio al commento di Zanato in LORENZO 1992); oltre a $\mathrm{ViBu} 2$ che ricorre dall'inizio (a partire dal $v$. 7; per vv. 7-9 cfr. ViBu 2, 3-5) fin quasi alla conclusione (per vv. I39-I42 cfr. ViBu 2, I9-22), con oltre una decina di riprese lungo tutto il testo, troviamo:

- l'apertura (le due terzine iniziali) che rimanda a Orazio (Epod. xv I-2 «Nox erat et caelo fulgebat Luna sereno / inter minora sidera») e (con le 
«saturate pecorelle» nella prima redazione) al Virgilio di un'altra bucolica ( ViBu го, 77: «saturae [...] capellae»);

- v. 2 I «ruminando forse erbe pallenti» che rimanda al Virgilio di un'altra bucolica (ViBu 6, 54 «pallentis ruminat herbas»);

- v. 62 «lento salcio» che rimanda al Virgilio di altre bucoliche ancora ( $V i B u$ 3, 83 e $V i B u$ 5, I6: «lenta salix»);

e altri echi che non sto a citare.

Come abbiamo visto accadere nell' Alberti, la conclusione, cambiando il contenuto didascalico/morale, si allontana dal modello latino. ${ }^{32} \mathrm{Mi}$ sembrano però significativi anche due rinvii alla terza egloga di Calpurnio. In $\mathrm{CaEc}$ 3, infatti, come nel Corinto, è riportato un canto/lamento ambientato (fatto inconsueto) in notturna: "Has tibi, Pbylli, precesiam pallidus, hos tibi cantus / dat Lycidas, quos nocte miser modulatur acerba» ( $\mathrm{CaEc} 3,45-46)$. Inoltre, da questa egloga sembrerebbe derivare (al di là dell'ascendenza virgiliana e ovidiana del personaggio) la descrizione che Corinto fa delle proprie ricchezze ai vv. I39-44:

S’io son ricco, tu il sai, ché in ogni lato

sonar senti le valle del mugito

de' buoi, e delle pecore il balato.

Latte ho fresco ad ognora, e nel fiorito

prato fragole, còlte belle e rosse,

pallide ove è il tuo viso colorito

(cfr. CaEc 3, 63-66: "Sum quoque divitior: certaverit ille tot haedos / pascere quot nostri numerantur vespere tauri. I Quid tibi, quae nosti, referam? Scis, optima Pbylli, / quam numerosa meis siccetur bucula mulctris / et quam multa suos suspendat ad ubera natos»). ${ }^{33}$

Vorrei infine segnalare un altro caso di doppio canto monodico in cui l'analisi della struttura consente di giungere a una migliore interpretazione del testo. Faccio riferimento alla terza egloga dell'Arcadia di Sannazaro ( $\mathrm{SaAr} 3 e$ ), che vede "Galicio solo» introdurre il canto di un pastore. L'egloga ha la forma di una canzone, di 6 stanze (di I3 vv. ciascuna) più congedo di $3 \mathrm{vv}$. Nella prima stanza (che fa da prologo), Galicio racconta di aver visto «un pastor, che [...] / cantava il terzo giorno / del mese inanzi aprile» (6-8). Dalla seconda stanza inizia il canto del pastore, che prima si rivolge ad Apollo-sole perché prolunghi il corso della giornata; poi (terza e quarta stanza) si rivolge a valli, piante 
e animali perché gli prestino ascolto e si rappacifichino, e a amorini, ninfe e fauni perché festeggino con lui; quindi, dal $v$. 53, nella quinta stanza, spiega la ragione della sua celebrazione: «In questo di giocondo nacque» la sua «Amaranta», il cui nome va incidendo su ogni faggio, cosi che, come precisa nella sesta stanza, «sempre fia noto il nome [...] di quella» di cui è innamorato (vv. 53, 62 e 75-77). Seguono i $3 v v$. del congedo, in cui si invita la canzone a pregare perché «quel di fausto et ameno [...] sia sempre sereno» (80-8I). Stando all'interpunzione proposta nelle moderne edizioni $i z \mathrm{v}$. del congedo fanno parte del canto del pastore, mentre secondo me, richiamandoci alle strutture chiuse, a cornice, di Virgilio (e non solo), quei $3 v v$. vanno attribuiti alla voce narrante di Galicio: se si presta attenzione ai dimostrativi, si nota che non si parla più di «questo dì del v. 53, ma di «quel dì citato ai $v v$. 7-8, e in effetti il congedo è quello della canzone di Galicio, non del canto del pastore. ${ }^{34}$

Concludendo, non è certamente un caso che questa analisi delle sovrapposizioni di diversi livelli (e della combinazione tra eventuale piano narrativo e messa in scena vera a propria) risulti cosi interessante e produttiva proprio in autori di cosi spiccate tendenze classicistiche. Ulteriori sondaggi più sistematici, e che magari includano anche il versanti latino quattrocentesco, ${ }^{35}$ consentiranno di tracciare un bilancio complessivo più definito.

Maria Finazzi 
Edizioni e commenti (segno con un * l'edizione da cui, se non altrimenti specificato, cito i testi):

Alberti i 966 = Leon Battista Alberti, Opere volgari, volume secondo, Rime $e$ trattati morali, a cura di C. Grayson, Bari, Laterza (Scrittori d'Italia, vol. 234)

Alberti i 97i = Leon Battista Alberti, Rime amorose e morali, stampato per i Cento amici del libro, a cura di G. Folena, Verona, Officina Bodoni

Alberti $1975 *=$ Leon Battista Alberti, Rime e versioni poetiche, edizione critica e commento a cura di G. Gorni, Milano-Napoli, Ricciardi

Alberti 2002 = Leon Battista Alberti, Rime / Poèmes, suivis de la Protesta / Protestation, Édition critique, introduction et notes par G. Gorni, Traduction de l'italien par M. Sabbatini, Paris, Les Belles Lettres

Arcadia $\mathrm{I} 888=$ Arcadia di Jacobo Sannazaro secondo $i$ manoscritti e le prime stampe, con note ed introduzione di M. Scherillo, Torino, Loescher

Arzocchi i995 = Francesco Arzocchi, Egloghe, edizione critica e commento a cura di S. Fornasiero, Bologna, Commissione per i testi di lingua

Boiardo $1996 *$ Matteo Maria Boiardo, Pastoralia, testo critico, commento e traduzione di S. Carrai, Padova, Antenore

Boiardo 2005 = Matteo Maria Boiardo, Pastorali, Introduzione di S. Carrai, Commento e Nota al testo di M. Riccucci, Milano-Parma, Fondazione Pietro Bembo-Guanda

Boiardo 20 io = Matteo Maria Boiardo, Pastoralia. Carmina. Epigrammata, a cura di S. Carrai e F. Tissoni, Scandiano, Centro Studi Matteo Maria Boiardo - Novara, Interlinea

Boiardo 20 i $5 *$ = Matteo Maria Boiardo, Pastorale. Carte de triomphi, a cura di C. Montagnani e A. Tissoni Benvenuti, Scandiano, Centro Studi Matteo Maria Boiardo - Novara, Interlinea

Calpurnio i 994 = Calpurnio Siculo, Egloghe, Introduzione, traduzione e note di M.A. Vinchesi, Milano, Rizzoli (ristampa 2010)

Calpurnio 2008 = T. Calpurnio Siculo, Eclogae, Introduzione, testo critico, traduzione e commento a cura di E. Di Lorenzo, Bruno Pellegrino, Napoli, Cuzzolin Calpurnio $2014^{*}=$ Calpurni siculi Eclogae, a cura di M.A. Vinchesi, Firenze, Le Monnier

Lorenzo $1992 *=$ Lorenzo De’ Medici, Opere, a cura di T. Zanato, Torino, Einaudi

Nemesiano $1997^{*}=$ Marco Aurelio Olimpio Nemesiano, Eclogae, a cura di G. Cupaiolo, Napoli, Loffredo

Sannazaro i 990 = Iacopo Sannazaro, Arcadia, a cura di F. Erspamer, Milano, Mursia (ristampa 2003)

Sannazaro $2013^{*}=$ Iacopo Sannazaro, Arcadia, Introduzione e commento di C. Vecce, Roma, Carocci 
SERVII grammatici $*=$ SERVII grammatici qui feruntur in Vergilii Bucolica et Georgica commentarii, recensuit Georgius Thilo, Lipsia, Teubner (rist. Hildesheim, Georg Olms, i 961)

Teocrito 2004 = Teocrito, Idilli e epigrammi, a cura di B.M. Palumbo Stracca, Milano, Rizzoli ( ${ }^{a}$ ed. I993)

Teocrito $2008 *$ = Teocrito, Idilli, a cura di M. Cavalli, in Callimaco, Epigrammi; Teocrito, Idilli; Meleagro, Epigrammi, Milano, Mondadori («I Classici Collezione»; I ${ }^{a}$ ed. Teocrito I99I)

Virgilio 2005 = Publio Virgilio Marone, Bucoliche, Note esegetiche e grammaticali a cura di M. Gioseffi, Milano, CUEM

Virgilio 2012 * = Publio Virgilio Marone, Le Bucoliche, Introduzione e commento di A. Cucchiarelli, Traduzione di A. Traina, Roma, Carocci

Studi:

Albonico 2017 = S. Albonico, Sulla struttura di alcune rime di Leon Battista Alberti, in Survivals, revivals, rinascenze. Studi in onore di Serena Romano, a cura di N. Bock, I. Foletti, M. Tomasi, Roma, Viella, pp. 379-90

Battera 1990 = F. Battera, L'edizione Miscomini (I482) delle Bucoliche elegantissimamente composte, in «Studi e problemi di critica testuale», 40, pp. I49-8 5

Cardini $2005 \mathrm{a}=\mathrm{R}$. Cardini, Alberti o della scrittura come mosaico, in Leon Battista Alberti pp. 9I-94 [già introduzione a R. Cardini, Mosaici. Il "nemico" dell'Alberti, Roma, Bulzoni, I 991]

Cardini 2005b = R. Cardini, Lo scaffale elegiaco dell'Alberti. Properzio e la "rifondazione" albertiana dell'elegia, in Leon Battista Alberti 2005, pp. I75-8 I

Carrai 2006 = S. Carrai, L'usignolo di Bembo. Un'idea della lirica italiana del Rinascimento, Roma, Carocci

Corfiati 2012 = C. Corfiati, Il «querulus fletus» della bella Amarillide, in Roma e il Papato nel Medioevo, Studi in onore di Massimo Miglio, II: Primi e tardi umanesimi: nomini, immagini, testi, Roma, Edizioni di Storia e Letteratura, pp. I4 I-46

Corfiati 2013 = C. Corfiati, Il fantasma di Teocrito: osservazioni sulla ricezione della bucolica greca nel Quattrocento, in «Cahiers de Recherches Médiévales et humanistes», 25, pp. $295-326$

De Robertis $198 \mathrm{I}=\mathrm{D}$. De Robertis, L'egloga volgare come segno di contraddizione, in «Metrica», II, pp. 6 I-80

Enciclopedia virgiliana $=$ Enciclopedia virgiliana, 5 voll., Roma, Treccani, I 984-I99

Leon Battista Alberti 2005 = Leon Battista Alberti. La biblioteca di un umanista, a cura di R. Cardini, con la collaborazione di L. Bertolini e M. Regoliosi, Firenze, Mandragora

Ponte 1978 = G. Ponte, recensione a AlberTi 1975, in «La rassegna della Letteratura italiana», LXXXII, pp. 536-59

Pretagostini $1980=\mathrm{R}$. Pretagostini, La struttura compositiva dei carmi teocritei, in «Quaderni Urbinati di Cultura Classica», 5, pp. 57-74

Tanturli I98 $_{\mathrm{I}}=$ G. Tanturli, Note alle rime dell'Alberti, in «Metrica», II, pp. I03-20 Tissoni Benvenuti $1978=$ recensione a ALBERTI I975, in «Studi medievali», XIX, pp. $1075-79$ 
von Albrecht 20 г 2 = M. von Albrecht, Virgilio. Un'introdu乏ione. Bucoliche - Georgiche - Eneide, Milano, Vita e Pensiero

Zanato 1998 = T. Zanato, Percorsi della bucolica laurenæiana, in La poesia pastorale nel Rinascimento, a cura di S. Carrai, Padova, Antenore, pp. 109-50

Presento qui i primi risultati di una analisi ancora parziale di testi bucolici quattrocenteschi, avviata in anni passati come progetto di dottorato presso l'Università di Losanna sotto la guida di Simone Albonico, e ripresa solo recentemente: ringrazio Massimo Danzi di avermi offerto l'occasione di parlarne al convegno di Ginevra sulla Poesia "en travesti". Ringrazio inoltre Federico Francucci per l'attenta rilettura di questo articolo e per la consulenza sul greco, e Simone Albonico per la revisione finale. Imprecisioni e inesattezze sono da attribuire esclusivamente a me. Per riferirmi ai testi bucolici utilizzerò il seguente sistema di sigle: $A l C_{0}=$ Alberti, Corymbus; AlTy = Alberti, Tyrsis; ArEg = Arzocchi, Egloghe; CaEc $=$ Calpurnio, Eclogae; BoPA = Boiardo, Pastoralia; BoPE = Boiardo, Pastorale; LoCo $=$ Lorenzo de Medici, Corinto; $N e E c=$ Nemesiano, Eclogae; $S a A r=$ Sannazaro, Arcadia $;$ TeId = Teocrito, Idilli; $V i B u=$ Virgilio, Bucoliche; in caso di raccolte di più testi (come le Bucoliche di Virgilio o le Pastorale del Boiardo), il numero della singola egloga è indicato con cifra araba dopo la sigla dell'opera (es. ViBu $\mathrm{I}=e c l$. I delle Bucoliche di Virgilio); solo per Sannazaro uso una "e" (che sta per egloga) dopo la cifra araba per fare riferimento alla porzione in versi all'interno della singola sezione in prosa ( $\mathrm{SaAr}$ ze = egloga contenuta nel terzo "capitolo" dell'Arcadia). Riporto qui i principali riferimenti bibliografici che mi sono stati utili per la stesura e la successiva revisione dell'intervento. Su struttura e diffusione nel Quattrocento degli Idilli di Teocrito cfr. Pretagostini r 980 e Corfiati 20I3; per i testi mi sono avvalsa delle edizioni commentate TeOcrito 2004 e TEOCRITO 2008. Sulle caratteristiche strutturali delle Bucoliche di Virgilio cfr. Enciclopedia virgiliana, s.v. Bucoliche, le edizioni commentate delle Bucoliche a cura di M. Gioseffi (Virgilio 2005) e a cura di A. Cucciarelli (Virgilio 20 i 2), e il volume von Albrecht 20 2. Su Calpurnio e Nemesiano cfr. le edizioni Calpurnio i 994, CaLpurnio 2008, Calpurnio 20i4 e Nemesiano i 997. Sulle egloghe dell'Alberti cfr. le edizioni delle rime di Grayson (Alberti i 966), Folena (Alberti i 97i) e Gorni (Alberti i975 e Alberti 2002), e il saggio Tanturli i98 I, gli studi di Roberto Cardini (Cardini 2005a e Cardini 2005b) e un recente articolo di Simone Albonico che ho potuto leggere in bozze (Albonico 2017), cui rimando per ulteriori osservazioni sulla struttura delle egloghe albertiane, in aggiunta a quelle da me avanzate qui. Sulla produzione bucolica del Boiardo cfr. le edizioni delle egloghe latine a cura di Carrai (BOIARDO I996 e BOIARDO 20IO) e delle egloghe volgari a cura di Marina Riccucci (Boiardo 2005) e a cura di Cristina Montagnani (Boiardo 2015). Per il Corinto di Lorenzo de' Medici cfr. l'edizione di Zanato (Lorenzo i992) e lo studio Zanato i 998. Per l'Arcadia di Sannazaro cfr. le edizioni di Scherillo (Arcadia i 888), Erspamer (Sannazaro i990) e Vecce (SannaZARO 2OI3).

I. Von Albrecht 201 2, pp. 47-48.

2. Carrai 2006, p. 29.

3. BoIARdo 2005, p. XIII. 
4. Carrai 2006, p. 29.

5. BOIARDO 2005, p. XVII.

6. Battera 1990.

7. VIRGILIO 2005 , p. I 52.

8. Von Albrecht 201 2, p. 6 I n.

9. A proposito del primo emistichio del v. I 3 Servio commenta «hortatur musas ad referenda ea, quae Silenus cantaverat pueris» (SERvII grammatici, p. 66).

io. Virgilio 2005 , p. 35 .

I I. Preciso che il commento di Servio lascia intendere che i vv. finali facciano parte del monologo di Coridone (dando ragione alla maggior parte degli editori); a proposito del v. 70 scrive infatti: «semiputata tibi f. v. i. ulmo est plus est, quam si inputata diceret; tolerabilius enim est non incipere aliquid, quam incepta deserere: hinc est illud in primo [Aen.] $\left.{ }_{37}\right\rangle$ mene incepto desistere victam? item in georgicis $<$ III 5 I9 $>$ atque opere in medio defixa reliquit aratra. sane geminam arguit neglegentiam, et quod semiputata vitis est, et quod frondosa ulmus; nam utrumque obest: unde est in georgicis $<\mathrm{II} 4 \mathrm{I} \mathrm{O}>$ bis vitibus ingruit umbra. et aut simpliciter intellegimus hunc locum, ut suam arguat neglegentiam: aut certe illud est: non mirum, me esse dementem, qui habeo vites semiputatas. nam in sacris dicitur, quod corripiatur furore qui sacrificaverit de vino, quod est de vitibus inputatis» (Servil grammatici, p. 28). Nella parte finale del commento di Servio la spiegazione «ut suam arguat neglegentiam» e la parafrasi «illud est: non mirum, me esse dementem, qui habeo», così formulate non possono che riferirsi alla voce del protagonista dell'egloga, e non del narratore. Von Albrecht 20 I 2, p. 2 I n., chiosa: «Si è pensato a un mutamento del personaggio parlante, tanto più che anche la parte introduttiva è pronunciata dall'autore in propria [sic] persona. I principali editori [...] pensano però ad autoallocuzione - quasi certamente a ragione, giacché pochi versi prima si tratta indubbiamente di apostrofe a se stesso».

I 2. Sintetizzo molto. A livello di "sottogeneri" (riprendendo la classificazione proposta da Von Albrecht 201 2) troviamo: dialogo (ViBu 1, ViBu 9), monologo ( $V i B u$ 2, ViBu 8 - ViBu 8 con verso intercalare) e gara canora (ViBu 3, ViBu s, $V i B u 7$ - ViBu 3 e $V i B u 7$ amebee); sono a vario titolo extra genere $V i B u$, ViBu 6 e $V i B u$ го. A livello di situazione abbiamo: due pastori in cammino o pastori in riposo che dialogano e/o si scambiano canti ( $V i B u$ I, ViBu 3, ViBu s, ViBu 9); un io narrante che riporta o introduce un carme ( $V i B u 4$, ViBu 6, ViBu 1o) o il canto di un pastore ( $\mathrm{ViBu}$ 2), o lo scambio di canti di due pastori ( $\mathrm{ViBu} 7, \mathrm{ViBu}$ 8). Secondo il numero delle voci che parlano o dialogano (che non sono quindi riportate) si va dal solo io narrante (come in $\mathrm{ViBu} 4$ e $\mathrm{ViBu}$ ro e secondo una delle possibili letture $V i B u$ 6) a un massimo di 3 interlocutori "reali", i cui discorsi, cioè, non sono riportati ma diretti (è il caso della gara poetica con giudice in $V i B u 3$ )

I 3. I casi di struttura aperta (vedi nota precedente) in Virgilio sono quindi uno $(\mathrm{ViBu} 8)$ o due (se in $V i B u 2$ attribuiamo $\mathrm{i} v \mathrm{vv}$. finali a Coridone e non all'io 
narrante). Un altro caso di struttura aperta si ha in $\mathrm{NeEc}_{4}$ in cui il testo finisce sull'ultima battuta dell'amebeo.

I4. Da questo punto di vista non condivido l'impaginazione del testo adottata dagli editori moderni: secondo me il verso finale non è da allineare con il primo verso delle terzine, come se fosse l'ultimo verso di chiusa di un capitolo ternario; si tratta invece dell'ultimo verso di una quartina (come notava anche Tanturli i 98 I, p. I I I n), da allineare perciò al terzultimo e al penultimo verso del testo; una conferma in tal senso viene anche dall'unica testimonianza arrivataci, il ms. $\mathrm{H}$ di mano del Feliciani (Cambridge MA, Houghton Library, ms. Typ. 24). Segnalo inoltre che nelle edizioni moderne gli ultimi $7 \mathrm{vv}$. del testo sono stati attribuiti a locutori diversi: i primi editori (Grayson e Folena) hanno attribuito le ultime due terzine (vv. 79-84) a Floro e la quartina finale a Tirsi (nonostante sul ms. manchi la didascalia per Tirsi); Gorni, rilevando la presenza di una didascalia non segnalata dal Grayson («phauni loquu(n)t(ur)» sul margine destro del manoscritto, all'altezza del v. 82), ha attribuito solo la penultima terzina a Floro e la terzina e la quartina finali a un diverso interlocutore collettivo (i Phauni). Su quest'ultima interpretazione può restare qualche dubbio, sia per la presenza di una «sgrammaticatura» (come notava Giovanni Ponte c'è un contrasto tra il soggetto plurale e il «vidi» del successivo v. 84 , cfr. Ponte 1978 , p. 538 ), sia per la posizione insolita della didascalia che farebbe pensare più a un'annotazione, come avviene in un altro luogo del ms., sempre con testo latino (a c. $67 \mathrm{r}$, accanto alla trascrizione del v. 25 di $\mathrm{ArEg}$ I, che recita: «La nocte aspecta pur che 'l giorno fazassi», si legge, su due righe: «ut extinguantur tenebre / et virtus in lucem appareat»).

I 5. Al pari di Alco e della Mirtia nel ms. Magl. VII i i4s ( $\left.\mathrm{F}_{\mathrm{ro}}\right)$, anche AlTy per l'argomento di tipo etico potrebbe essere denominato "morale".

I6. Cardini 2005a e Cardini 2005b.

17. Questo è un dettaglio che non trovo evidenziato nei commenti alle Pastorale, anche se è menzionato nell'introduzione di Carrai a $B o P A 8$, a cui comunque tutti i commenti rinviano: Carrai sottolinea che la misura dei canti dell'egloga latina di Boiardo riprende quella del primo canto, di Damone, nel modello virgiliano ViBu 8 (Boiardo i 996, p. 79); i due canti della Pharmacentria sono infatti di 45 vv. il primo e di $46 \mathrm{vv}$. il secondo - per inciso: da Geymonat proviene la proposta di ricondurli entrambi alla stessa lunghezza non aggiungendo un v. intercalare al primo, dopo il v. 28 , bensì omettendo l'intercalare del v. 76 dal secondo.

I 8. BOIARDO 2OI 5, p. 20 I.

I9. Von Albrecht 201 2, p. 2 I.

20. Forse è solo una coincidenza che nel testo virgiliano sia individuabile una struttura interna di 5 blocchi (scanditi da esclamazioni e vocativi) di I I, I I, I 7, I I e I3/r $8 \mathrm{vv}$, e che nel testo albertiano la ballata distribuisca il testo della parte centrale in 5 blocchi ( 5 stanze di $8 \mathrm{vv}$, I I se si somma la ripresa di 4 vv; più un sesto blocco in terzine di $\mathrm{I} 2 \mathrm{vv}$ ). 
2 I. Vedi nota i 5 . Secondo Gorni, «l'incertitude que la tradition manuscrite affiche à l'égard de la définition du genre littéraire de ce poème n'est que le symptôme des virtualités de la poésie d'Alberti, expérimentale au plus haut degré» (ALBERTI 2002, p. 194).

\section{Alberti i 975 , p. 70.}

23. In $V i B u$ I, 45 così viene apostrofato, insieme ad altri postulanti (tra cui evidentemente degli schiavi), Titiro, giunto al cospetto del deus romano: «pascite ut ante boves, pueri; summittite tauros»; e in ViBu 6, I 3-I 4 Chromis et Mnasyllos che assalgono Silenum sono due pueri, stavolta nel senso di giovani o di giovani pastorelli (riprendo queste informazioni dai commenti alle Bucoliche di Gioseffi e di Cucchiarelli).

24. Tissoni Benvenuti i978, p. 1078 .

25. De Robertis 1981, pp. 71-72.

26. Alberti 2002, p. ios.

27. Con un gioco di scambi e incroci già ampiamente evidenziato dalla critica.

28. A parte la minima traccia rimasta nella didascalia.

29. La stessa cosa succede in TeId 3, dove il capraio presenta se stesso prima nell'atto di fare una serenata alla sua Amarilli (con un canto tutto pervaso di «contenuti attuali»), e poi di eseguire un canto «intessuto su contenuti mitologici» (Pretagostini i 980, p. 60). La didascalia di BoPE 9 non lascia dubbi riguardo al carattere monodico dell'egloga; in caso contrario ci sarebbe stata un'indicazione della presenza del poeta sulla scena, in qualità di narratore, come avviene in $B o P E$ 2, $B o P E E_{3}$ е $B o P E$ ı. Queste, per intero, le didascalie delle quattro egloghe boiardesche: «Egloga secunda ne la qual parla lo auttor e canta Galathea» (BoPE 2); «Ne la terza parla lo auttore; cantano alternatamente nello amabeo rispondendosi Aristeo e Daphnyde» (BoPE 3); «In questa nona lamentasi cantando Corydone per la sua cara Nysa a Mopso maritata» (BoPE 9); «Ne l'ultima parla lo auttore; canta Orpheo el panagyrico de lo incomperabile signor duca de Calabria» (BoPE ıо).

30. Zanato I998, pp. i I4-i 5 , suggerisce invece l'intenzione laurenziana di ricalcare l'arzocchiano Terinto/Tirinto, con il «ricorso al nome di una città greca»; le due cose non si escludono.

3 I. La prima redazione ci è «giunta frammentaria attraverso la stampa ottocentesca delle Opere laurenziane curata dalla Crusca (Molini, Firenze i 825 )»: secondo Zanato, «è la (probabile) stesura autografa dei primi versi del Corinto, che rappresenta l'originaria redazione dell'egloga, poi in alcuni punti [...] modificata» (LORENZO I992, p. I40).

32. Un allontanamento che in Lorenzo è segnalato anche dall'uso insistito della rima sdrucciola proprio nella parte finale del testo. 
33. Aggiungo che da $\mathrm{CaEc}_{4}, 3 \mathrm{I}-32$ «spes magis arridet. Certe ne fraga rubosque / colligerem» provengono forse le «fragole» del v. I43 di LoCo. La «presenza [...] non [...] consueta» di fraga è segnalata anche nel Querulus fletus di Paracleto Malvezzi da Corfiati 2012 , p. I42.

34. Per inciso, nell'edizione Scherillo (Arcadia I 888) la questione non si pone visto che non si fa uso di virgolette o altra interpunzione per distinguere le diverse voci.

35. Intanto, uno sguardo orientato alle strutture mi porta a proporre una diversa interpretazione dei due stadi relazionali di BoPA 3: la redazione di 8 egloghe tradita dall'edizione $G$ (nel complesso da considerarsi come abbozzi delle singole egloghe) per questo testo presenta meno differenze rispetto alla redazione definitiva; in particolare, la redazione $G$ presenta solo 99 vv., e viene regolarizzata da Boiardo con un'aggiunta al v. ig (che segnalo in corsivo), che porta la seconda battuta di Poeman da 3 (vv. I7-19 di G) a 4 versi (vv. I7-20):

abbozzo $(G)$

red. definitiva

P. Horrida diffugiunt glacialis tempora brumae P. Horrida diffugiunt glacialis tempora brumae et nivibus positis placidi mitescere montes et nivibus positis placidi mitescere montes incipiunt, incipiam pariter cantare Citherym. incipiunt, pariter solitus mibi crescere fervor incipit: incipiam pariter cantare Cytherim.

Considerato che tale battuta è la prima dello scambio amebeo tra Poeman e Silvano, che poi prosegue anche nell'abbozzo con i 5 battute di 4 vv. ciascuna, mi pare più opportuno ipotizzare che la redazione attestata da $G$ fosse già di ı oo vv. e che la testimonianza di $G$ sia semplicemente lacunosa a causa di un saut du même au même; la stessa battuta di Poeman mi pare assumere un senso compiuto solo con l'integrazione della porzione "caduta" (che segnalo in corsivo) nella trascrizione di G: «Fugge la stagione invernale irta di ghiaccio e, spogliatisi della neve, cominciano a scaldarsi i quieti monti; cosi comincia a crescere il mio consueto fervore ed io così inizierò a celebrare Citeride». 